\title{
Estudio de las estrategias de resolución de conflictos y habilidades sociales de alumnas y alumnos mediadores en educación secundaria obligatoria
}

\author{
DOI: $10.46981 /$ sjhv2n1-007
}

Received in: November 1st, 2020

Accepted in: December 30th, 2020

\author{
Isabel Silva Lorente \\ Associate Professor \\ Institution: Centro Universitario Cardenal Cisneros (University of Alcala) \\ Address: Av. Jesuitas, 3428806 Alcalá de Henares, Madrid, Spain. \\ E-mail: isabel.silva@ cardenalcisneros.es
}

\section{Juan Carlos Torrego Seijo}

Associate Professor

Institution: University of Alcala

Address: Aulario María de Guzmán, C/ San Cirilo s/n. 28801, Alcalá de Henares, Madrid, Spain

E-mail: juancarlos.torrego@uah.es

\section{Ph.D. Elena M. Lorenzo-Llamas}

Adjunt Professor of Research and Diagnostic Methods in Education field (MIDE)

Institution: University of Alcalá

Address: Aulario María de Guzmán, C/ San Cirilo s/n. 28801, Alcalá de Henares, Madrid, Spain.

E-mail: elenam.lorenzo@uah.es

\section{RESUMEN}

Al revisar investigaciones y trabajos previos relacionados con la mediación escolar, se puede observar que una amplia mayoría ofrece materiales de carácter práctico acerca de cómo llevar a cabo una mediación. Es menor el número de trabajos que evalúan el impacto que tienen este tipo de programas sobre la vida del centro y sobre los miembros que forman parte de la comunidad educativa. Así, el objetivo de esta investigación es analizar el impacto que tiene un programa de mediación entre iguales que se ha llevado a cabo en un IES (Instituto de Educación Secundaria) de la Comunidad de Madrid España- con alumnos y alumnas de $3^{\circ}$ y $4^{\circ}$ de la ESO que actuaron como mediadores. Se persigue conocer qué habilidades sociales adquieren y si mejoran sus herramientas para resolver conflictos. Para ello, se ha utilizado un diseño cuasi-experimental pretest-postest con grupo de control no equivalente. Los resultados del estudio muestran que hay diferencias significativas en algunas de las habilidades sociales medidas, si comparamos los grupos control y experimental. Asimismo, la pertenencia a un equipo de mediación también está relacionado con el desarrollo de algunas habilidades sociales y de resolución de conflictos.

Palabras clave: convivencia pacífica, enseñanza secundaria, mediación, resolución de conflictos. 


\section{INTRODUCCIÓN}

Si fijamos la mirada en nuestros centros educativos, podemos observar cómo desde hace unos años se está produciendo un aumento de la sensibilidad social ante la necesidad de construir una convivencia pacífica. Además, se abre paso un nuevo enfoque a la hora de gestionar el conflicto en contextos educativos: la mediación (Avilés Martínez, \& Alonso Elvira, 2017; Pinto, Torrego \& Martins, 2016).

La sociedad en la que vivimos demanda aprendizajes relacionados con la participación, la cooperación o la tolerancia; disponer de habilidades sociales, de solidaridad o aprender a gestionar pacíficamente los conflictos interpersonales son virtudes propias de una cultura de convivencia (GarcíaRaga \& López, 2011) y deben estar presentes en el proceso de enseñanza aprendizaje. Como destaca Monjas (2010) este tipo de habilidades permiten a las personas convivir y relacionarse con otros de una manera adecuada y eficaz.

Estos aprendizajes no se refieren únicamente a contenidos teóricos, se trata de habilidades que se logran, como reflejan Trianes, Muñoz y Jiménez (1997) a través de la experiencia directa en los distintos contextos en los que se mueve la persona y en la educación explícita de este tipo de habilidades. Si hablamos concretamente de la mediación, participar en este tipo de procesos tiene un impacto positivo en las habilidades sociales de los alumnos y alumnas, entre otras por dos razones: por un lado, el propio proceso de mediación exige poner en marcha distintas habilidades sociales: escucha, empatía, negociación, etc, pero además, la formación que recibe un estudiante o cualquier otra persona que entra a formar parte del equipo de mediación, implica también la puesta en práctica y el entrenamiento de distintas habilidades: afectivas, comunicativas, interpersonales, etc.

Si retrocedemos hasta el origen de la mediación escolar, encontramos sus inicios en los años 70 cuando en Estados Unidos comienza a utilizarse la mediación en el ámbito educativo. En España, es en los años 90 cuando en el País Vasco se desarrolla un programa pionero en mediación escolar desde el Centro de Investigación para la Paz Gernika Gogoratuz (Uranga, 1997). Desde los años 90, la utilización de la mediación como herramienta de mejora de la convivencia ha ido en aumento, convirtiéndose en un instrumento que, además de permitir una adecuada gestión de los conflictos, dota al alumnado de competencias socioemocionales y les prepara para el ejercicio de una ciudadanía activa.

Boqué (2018) define la mediación como un proceso formal de gestión pacífica de conflictos en el cual participan activamente las personas implicadas en el mismo junto con otra tercera persona externa que los acompaña en la exploración del conflicto, en el proceso de comunicación entre ambas y en la cooperación para buscar un acuerdo satisfactorio para ambas partes y pactado desde la libertad.

La idea básica para explicar qué supone un programa de mediación es que un grupo de alumnos y alumnos (o profesores, familias y otros miembros de la comunidad educativa) se forman y se hacen 
expertos en la utilización de la mediación en caso de que se produzca un conflicto. Cualquier persona, alumno/a, profesores o PAS, involucrado en un conflicto o disputa puede solicitar la utilización de la mediación para llegar a un acuerdo. De este modo, se convierte en una alternativa al sistema basado en las sanciones que las partes en conflicto pueden escoger voluntariamente. Habitualmente las personas mediadoras intervienen en situaciones como conflictos entre iguales, malentendidos, disputas, confrontación de intereses, etc. (Avilés, 2017).

Como señalan García-Raga, Grau \& López-Martín (2017), la mediación, además de ser un proceso que permite afrontar los conflictos de un modo participativo, permite que las personas involucradas aprendan diferentes habilidades sociales y personales, y en definitiva facilita el fortalecimiento de las relaciones interpersonales entre el alumnado y favorece la convivencia escolar.

Si nos centramos en los beneficios que se producen sobre el alumnado, podríamos señalar que tener amigos o el apoyo de algún compañero previene verse inmerso en una situación de agresión. Además, este tipo de relaciones puede dar la oportunidad de involucrarse en una relación no basada en la violencia y puede ayudar a aquellos alumnos y alumnas con más dificultades en sus relaciones, al promover un contexto nuevo en el que poder entrenar o poner en práctica distintas habilidades sociales (Ortega-Ruiz \& del Rey, 2004).

Distintas investigaciones (Guiral, Badenes \& Adrián, 2016; Ibarrola-García \& Iriarte, 2014a; García-Raga, Sanchis, Mora \& Santana, 2016; Boqué, 2018; Usó, Villanueva \& Adrián, 2016) recogen el impacto positivo que tiene la mediación en el contexto escolar. Una de las áreas que ha recibido mayor atención es la que tiene que ver con las habilidades sociales del alumnado; en este sentido, algunas investigaciones muestran que se produce un incremento de la empatía; por ejemplo, Ibarrola-García \& Iriarte (2013) muestran como los mediadores, tanto alumnos como profesores, coinciden en señalar la empatía como el principal aprendizaje emocional que se realiza en este tipo de procesos. También se destaca cómo la mediación ayuda a ser consciente del papel que tienen las emociones sobre la conducta de las personas. En esta línea, Cassinerio \& Lane-Garon (2006) concluyen en su investigación que los programas de mediación en contexto educativo mejoran el comportamiento altruista del alumnado.

Disponemos de estudios sobre los beneficios, las dinámicas, las estructuras y el impacto de los programas desde la perspectiva de los alumnos y de los profesores (Grau, García-Raga \& López-Martín, 2016; Ibarrola-García \& Iriarte, 2014b; Silva \& Torrego, 2017), así como sobre los fenómenos perturbadores de la convivencia escolar, por ejemplo, la violencia entre pares (Ortega-Ruiz, del Rey \& Casas, 2016), sobre los resultados de la intervención de los equipos de mediación escolar (Pinto, Torrego \& Martins, 2016), la satisfacción de los alumnos participantes en sesiones de mediación y acerca del clima social de la escuela (Peñalva, López, Vega \& Satrústegui, 2015). A pesar de estos datos, todavía 
son escasos los estudios empíricos que estudian el impacto de la mediación escolar (García-Raga, Grau \& López-Martín, 2017).

También en los últimos años se está realizando un esfuerzo por recoger la mediación escolar dentro de la normativa nacional y autonómica, apareciendo por primera vez en la Ley 27/2005, de 30 de noviembre de fomento de la educación y la cultura de la paz. Lo que se puede encontrar en la legislación son referencias escuetas a su interés e importancia, sin que haya habido un desarrollo adecuado de cómo implementar este tipo de programas en la realidad educativa.

Usó, Villanueva \& Adrián (2016) señalaban recientemente que los estudios que se han llevado a cabo sobre la mediación escolar son contradictorios. Destacan como una posible explicación de estos resultados las diferencias metodológicas ya que algunos estudios se basan en las impresiones personales de los participantes mientras que otros han realizado evaluaciones más sistemáticas. Asimismo, en el Informe 2016 sobre el Estado Educativo Español (Consejo Escolar del Estado, 2016) también se recoge la necesidad de un estudio empírico para detectar qué prácticas contribuyen a crear un clima de respeto y trabajo en el centro.

Como respuesta a esta demanda, consideramos necesario profundizar en el análisis de este tipo de programas. Como elemento diferenciador, proponemos en esta investigación la utilización de herramientas estandarizadas de evaluación de las habilidades sociales que pueden contribuir a una evaluación más objetiva de estas habilidades, ya que la mayoría de las investigaciones de este tipo utilizan cuestionarios elaborados ad hoc. Así, esta investigación, se centra en la evaluación de las habilidades sociales de un grupo de alumnos que entra a formar parte por primera vez del equipo de mediación de un Instituto de Educación Secundaria (IES) de la Comunidad de Madrid. Pretender evaluar si existe un impacto en las estrategias de resolución de conflicto y en las habilidades sociales de un grupo de estudiantes que han participado durante un curso académico en el programa de mediación en comparación con un grupo control.

\section{MÉTODO}

\subsection{OBJETIVOS}

El objetivo de esta investigación es comparar el aprendizaje de habilidades sociales que adquiere el grupo experimental, formado por los alumnos y alumnas de $3^{\circ}$ y $4^{\circ}$ de Educación Secundaria Obligatoria que son seleccionados para participar en el equipo de mediación entre iguales en un instituto, en comparación con un grupo control. 


\subsection{PARTICIPANTES}

En esta investigación han participado un total de 33 alumnos y alumnas (15 en el grupo experimental y 18 en el grupo control). Para formar el grupo experimental se seleccionaron aquellos alumnos y alumnas que se incorporaban por primera vez al equipo de mediación, ya que lo que se pretendía era evaluar si se producía una mejora o no de las estrategias y habilidades tras un año de participación en el programa de mediación. Para realizar la selección del alumnado se tuvieron en cuenta los siguientes criterios: como mínimo se debería haber realizado una mediación formal en el curso académico, haber participado en una formación inicial específica sobre mediación de 8 horas y haber asistido a lo largo del curso escolar a las reuniones mensuales del equipo de mediación.

El grupo control lo conforman un total de 18 estudiantes del mismo instituto que ni este curso ni cursos previos, ha formado parte del proyecto de mediación del centro. Las edades de los alumnos y alumnas está en torno a los 14 años: la media de edad es muy similar si comparamos el grupo experimental $(M=14,47 \mathrm{DT}=, 640)$ y el grupo control $(\mathrm{M}=14,44 \mathrm{DT}=, 784)$. En la Tabla I, mostramos el número de participantes divididos por grupos y en función del sexo.

Tabla 1.

\begin{tabular}{ccccc}
\multicolumn{5}{c}{ Alumnado participante } \\
Gr. Control & Gr. Experimental & TOTAL \\
\hline Mujeres & Hombres & Mujeres & Hombres \\
7 & 11 & 4 & 11 & 33 \\
\hline
\end{tabular}

Para la realización de esta investigación, se informó a los participantes y a las familias sobre el proceso de evaluación y los objetivos de la investigación y se les solicitó el consentimiento informado para participar en el estudio.

\subsection{METODOLOGÍA}

Con objeto de explicar la máxima información posible, se ha utilizado un diseño cuasiexperimental pretest-postest con grupo de control no equivalente. Se opta por este diseño, porque no se consideraba conveniente realizar una selección aleatoria de los individuos de la muestra, sino una selección intencional acorde a los objetivos del estudio.

Todo el alumnado que participa en el estudio, ya sea del grupo control o del experimental, tuvo que responder a los siguientes cuestionarios: la Escala de afrontamiento para adolescentes (ACS) (Frydenberg y Lewis, 1996) y la Escala de habilidades sociales (EHS) (Gismero, 2000). Estas dos escalas se pasaron en dos momentos diferentes del año: en el mes de septiembre, antes del inicio del programa, y en el mes de junio; el objetivo perseguido era evaluar si se producía alguna diferencia entre la evaluación pre y postest. 
El análisis de datos ha tenido por finalidad realizar las siguientes comparaciones:

- En la evaluación postest se ha realizado una comparación de medias entre el grupo control y el experimental tanto de las habilidades sociales del alumnado como de sus herramientas para resolver conflictos, medidas a través a través de los dos instrumentos de evaluación ya señalados.

- Tomando únicamente la muestra del grupo experimental, se ha realizado una comparación pretest y postest para observar si existe una mejora de las habilidades sociales entre ambos momentos temporales.

Mediante el programa SPSS v.25.0 se ha realizado un análisis estadístico descriptivo: se describen los datos empíricos a través de las medidas de tendencia central, principalmente la media. Se recogen asimismo medidas de dispersión, que quedan reflejadas a través de los datos sobre la desviación típica. Además de este análisis, se ha utilizado la prueba $t$ de Student para realizar un contraste de medias, comparando los grupos del cuasi-experimento y para valorar si existían diferencias significativas. Asimismo, se ha medido el tamaño del efecto del programa de mediación utilizando el cálculo del estadístico $d$ de Cohen entre el grupo experimental y el control.

\subsection{INSTRUMENTOS}

Los instrumentos de evaluación utilizados en esta investigación han sido los siguientes:

- Escala de afrontamiento para adolescentes (ACS) (Frydenberg \& Lewis, 1996), validada para población española y publicada por el grupo TEA. Se trata de un inventario de autoinforme compuesto por 80 ítems, 79 con opción de respuesta cerrada y 1 de respuesta abierta, que se puntúan mediante una escala de tipo Likert de grado 5. Dicha escala abarca desde $\mathrm{A}=$ "No se me ocurre nunca o no lo hago" hasta $\mathrm{E}=$ "Me ocurre o lo hago con mucha frecuencia". El último ítem es una pregunta abierta acerca de otras estrategias que utiliza el adolescente para enfrentarse a sus problemas. Dicha escala obtiene puntuaciones directas en las estrategias de afrontamiento, entendidas estas como acciones concretas que el individuo realiza para enfrentarse a la situación. Las 18 estrategias de afrontamiento valoradas son: Buscar apoyo social (1); Concentrarse en resolver el problema (2); Esforzarse y tener éxito (3); Preocuparse (4); Invertir en amigos íntimos (5); Buscar pertenencia (6); Hacerse ilusiones (7); Falta de afrontamiento o no afrontamiento (8); Reducción de la tensión (9); Acción social (10); Ignorar el problema (11); Autoinculparse (12); Reservarlo para sí (13); Buscar apoyo espiritual (14); Fijarse en lo positivo (15); Buscar ayuda profesional (16); Buscar diversiones relajantes (17); y, Distracción física (18). 
- Escala de habilidades sociales (EHS) de Gismero González (2000). Es un cuestionario compuesto por 33 ítems que exploran la conducta habitual del individuo en situaciones concretas y valora hasta qué punto las habilidades sociales modulan estas actitudes. La muestra empleada para el análisis correlacional fue llevada a cabo con 1015 jóvenes, todos los índices de correlación superan los factores encontrados en el análisis factorial de los elementos en la población general. La EHS de Gismero muestra una consistencia interna alta como se expresa en su coeficiente de confiabilidad $\alpha=0,88$. Utiliza una escala tipo Likert de grado cuatro, desde $\mathrm{A}=$ "No me identifico en absoluto; la mayoría de las veces no me ocurre o no lo haría" hasta D = "Muy de acuerdo y me sentiría o actuaría así en la mayoría de los casos". Aporta como resultado un Índice Global del nivel de habilidades sociales o aserción de los individuos analizados y detecta cuáles son sus áreas más problemáticas respecto a dicha asertividad. Las habilidades sociales valoradas con EHS se sintetizan en 6 subescalas: Autoexpresión en situaciones sociales (I); Defensa de los propios derechos como consumidor (II); Expresión de enfado o disconformidad (III); Decir "no" y cortar interacciones (IV); Hacer peticiones (V); e Iniciar interacciones positivas con el sexo opuesto (VI).

\section{RESULTADOS}

Comenzamos mostrando los resultados que arroja la escala ACS en las 18 estrategias de afrontamiento valoradas en los adolescentes para resolver conflictos. En la tabla 2, se puede observar en la columna que refleja la media, que la mayoría de estrategias son utilizadas de manera más frecuente por el grupo experimental en comparación con el grupo control; es decir, en la evaluación realizada al final el curso escolar, aquellos alumnos y alumnas que han formado parte del equipo de mediación manifiestan a través de sus respuestas a la escala ACS que utilizan en mayor grado las distintas herramientas para resolver conflictos en comparación con el grupo de estudiantes que no ha formado parte del equipo de mediación.

Se ha evaluado si se producen diferencias significativas entre los grupos experimental y control utilizando la prueba $t$ de Student para muestras independientes. Como se puede observar en la Tabla 2, se produce una diferencia de medias significativa en cuatro de las estrategias medidas, valoradas al 95\% de margen de confianza * $(p \leq, 05)$ e incluso al 99\% de intervalo de confianza ${ }^{* *}(p \leq, 01)$ son: "Buscar apoyo social” $\left(t(31)=3,433^{* *} p=, 002\right)$, “Concentrarse en resolver el problema” $\left(t(31)=2,291{ }^{*} p=, 029\right)$, "Buscar ayuda profesional" $\left(t(31)=3,124{ }^{* *} p=, 004\right)$ y "Buscar diversiones relajantes" $(t(31)=2,081$ $\left.{ }^{*} p=, 046\right)$. 
En cuanto al tamaño del efecto, considerando que la $d$ de Cohen representa el número de desviaciones típicas que separan al grupo experimental del grupo control en la variable evaluada con el ACS, los resultados significativos indican una diferencia de: una desviación típica y media en Buscar apoyo social (1); más de media desviación típica en Concentrarse en resolver el problema (2); sobrepasa una desviación típica en Buscar ayuda profesional (16); y, más de media desviación típica en Buscar diversiones relajantes (17). Mayoritariamente con un tamaño del efecto grande $(d \geq .80)$.

Tabla 2.

Resultados de la Prueba t de Student para muestras independientes en la escala ACS.

\begin{tabular}{|c|c|c|c|c|c|c|c|}
\hline & \multicolumn{2}{|c|}{ Grupo Experimental } & \multirow{2}{*}{$\frac{\text { Grupo }}{\mathrm{M}}$} & \multirow{2}{*}{$\frac{\text { Control }}{\text { DT }}$} & \multirow[b]{2}{*}{$t(31)$} & \multirow[b]{2}{*}{$p$} & \multirow[b]{2}{*}{$d$ Cohen } \\
\hline & $\mathrm{M}$ & DT & & & & & \\
\hline 1. Buscar apoyo social & 77,07 & 12,69 & 61,33 & 13,44 & 3,43 &, $002 * *$ & 1,45 \\
\hline $\begin{array}{l}\text { 2. Concentrarse en } \\
\text { resolver el problema }\end{array}$ & 76,27 & 14,62 & 65,33 & 12,80 & 2,29 &, $029^{*}$ &, 80 \\
\hline 3. Esforzarse y tener éxito & 70,40 & 8,39 & 64,22 & 14,36 & 1,47 & 152 &, 53 \\
\hline 4. Preocuparse & 72,40 & 19,86 & 68,44 & 14,64 &, 66 &, 515 &, 23 \\
\hline $\begin{array}{l}\text { 5. Invertir en amigos } \\
\text { íntimos }\end{array}$ & 64,27 & 12,69 & 64,44 & 13,51 &,- 04 & ,969 &,- 01 \\
\hline 6. Buscar pertenencia & 70,67 & 7,20 & 66,89 & 12,49 & 1,04 & ,308 &, 37 \\
\hline 7. Hacerse ilusiones & 55,20 & 14,60 & 56,22 & 11,10 &,- 23 & ,821 &,- 08 \\
\hline 8. Falta de afrontamiento & 42,93 & 10,19 & 47,78 & 12,91 & $-1,18$ & ,248 &,- 42 \\
\hline 9. Reducción de la tensión & 48,80 & 18,41 & 44,67 & 12,89 &, 76 & ,455 &, 26 \\
\hline 10. Acción social & 42,33 & 14,00 & 42,50 & 13,64 &,- 04 & ,973 &,- 01 \\
\hline 11. Ignorar el problema & 43,00 & 14,74 & 44,72 & 12,06 &,- 37 & ,714 &,- 13 \\
\hline 12. Autoinculparse & 51,67 & 13,97 & 47,28 & 8,67 & 1,10 & ,278 &, 65 \\
\hline 13. Reservarlo para sí & 53,33 & 13,72 & 49,44 & 12,82 &, 84 & ,407 & 29 \\
\hline $\begin{array}{l}\text { 14. Buscar apoyo } \\
\text { espiritual }\end{array}$ & 41,33 & 10,08 & 41,94 & 12,62 &,- 15 & 881 &,- 05 \\
\hline 15. Fijarse en lo positivo & 65,67 & 10,50 & 60,56 & 12,59 & 1,25 &, 221 &, 44 \\
\hline $\begin{array}{l}\text { 16. Buscar ayuda } \\
\text { profesional }\end{array}$ & 62,33 & 15,80 & 47,78 & 10,88 & 3,124 &, $004 * *$ & 1,07 \\
\hline $\begin{array}{l}\text { 17. Buscar diversiones } \\
\text { relajantes }\end{array}$ & 80,27 & 11,79 & 70,39 & 14,89 & 2,08 &, $046^{*}$ & ,74 \\
\hline 18. Distracción física & 72,80 & 17,71 & 69,22 & 23,14 & ,491 & ,627 &, 17 \\
\hline
\end{tabular}

Nota: Se asume igualdad de varianzas en la prueba de Levene. * $p \leq, 05 ; * * \leq, 01 ; * * * p \leq, 001$.

$\mathrm{Al}$ analizar las cuatro estrategias en las que hay diferencias significativas, se puede encontrar relación entre las tres primeras estrategias y los contenidos trabajados, tanto en la formación recibida por los alumnos mediadores, como en el propio proceso de mediación que realiza el alumnado cuando alguien demanda este servicio; la última estrategia, Buscar diversiones relajantes (17) es un tipo de estrategia que parece más complicado relacionar con los contenidos trabajados en la formación en mediación.

La primera estrategia: Buscar apoyo social (1) es una herramienta que está vinculada con la capacidad de relacionarse con otras personas, se relaciona también con la capacidad de expresar los propios sentimientos, una habilidad con gran utilidad en las relaciones interpersonales. Se considera que 
la participación en procesos de mediación puede impulsar estas habilidades. Si nos centramos en la segunda estrategia: Concentrarse en resolver el problema (2), se trata de una habilidad que presupone poder dejar a un lado pensamientos y sentimientos que no contribuyen a la resolución del problema y centrar los recursos en aspectos que sí pueden ser útiles. En este sentido, un aspecto que sí se trabaja en la formación que reciben los alumnos mediadores está relacionada con aprender a seleccionar los aspectos nucleares del conflicto, desechando aquellos más superficiales. La tercera estrategia, Buscar ayuda profesional (16) es una habilidad que implica la capacidad de acudir a alguien más experto para buscar consejo; este tipo de habilidades se refuerzan a través de este tipo de programas y estructuras, en cuanto que promueven las relaciones entre los alumnos y alumnas y los distintos profesionales del centro que tienen un mayor bagaje y experiencia en la gestión del conflicto: orientadores, Profesor Técnico de Servicios a la Comunidad (PTSC) o tutores de mediación. Aunque existen otras variables incontroladas que pueden estar influyendo en la relación causal, se podría pensar que formar parte de una estructura de alumnos mediadores permite el desarrollo de algunas de las habilidades citadas.

A continuación, nos centramos en analizar los resultados pretest y postest del grupo experimental en las 18 estrategias medidas con la prueba ACS ya señalada. Como se puede observar en la Figura 1, una gran parte de las estrategias medidas son utilizadas más frecuentemente en el postest, o lo que es lo mismo, hay una mayor utilización de estas estrategias de afrontamiento de conflictos al finalizar el curso, una vez que el alumnado ha participado durante un año en el equipo de mediación. Para interpretar la Figura 1, el intervalo de los valores mostrados va de los 20 puntos, que implica que no se utiliza la estrategia, hasta 105 puntos, que indicaría mucha frecuencia.

Figura 1. Media de las estrategias del ACS del grupo experimental en la evaluación pretest y postest

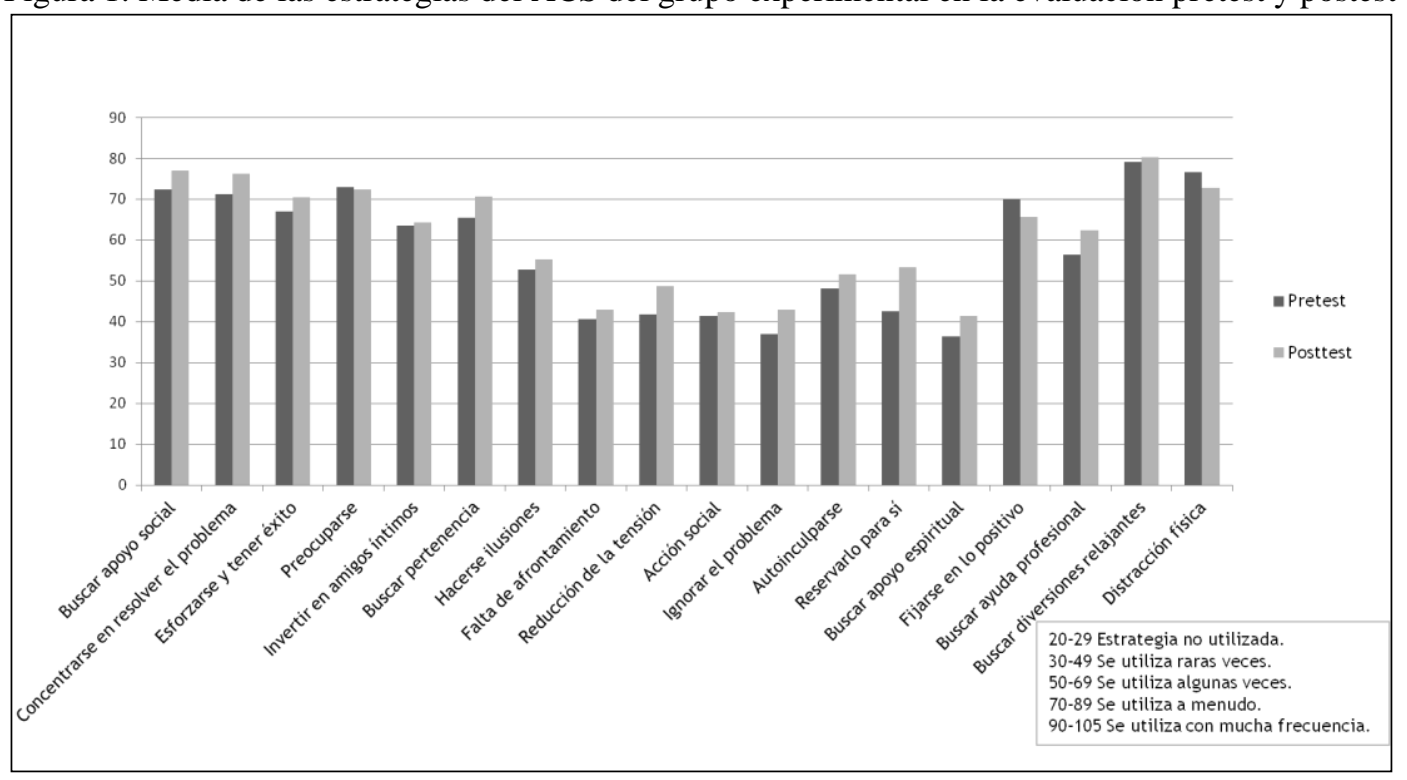


Los datos parecen apoyar la efectividad de este programa de mediación, en cuanto que hay, en general, una mayor utilización de estas estrategias en la evaluación postest, sin embargo, cabe señalar que, atendiendo a los resultados de la prueba $t$ de Student, no se han producido diferencias significativas al comparar los resultados pre y postest, tal y como queda reflejado en la Tabla 3.

Tabla 3.

Resultados de la Prueba t de Student para muestras relacionadas en la escala ACS.

\begin{tabular}{lrrrr}
\hline & M & DT & $t(14)$ & $p$ \\
\hline 1. Buscar apoyo social & $-2,67$ & 16,40 &,- 63 &, 539 \\
2. Concentrarse en resolver el problema & $-2,93$ & 17,40 &,- 65 &, 524 \\
3. Esforzarse y tener éxito & $-3,47$ & 10,01 & $-1,34$ &, 201 \\
4. Preocuparse & 2,53 & 20,12 &, 49 &, 633 \\
5. Invertir en amigos íntimos &, 53 & 9,55 &, 22 &, 832 \\
6. Buscar pertenencia & $-5,33$ & 14,71 & $-1,40$ &, 182 \\
7. Hacerse ilusiones & $-1,33$ & 13,99 &,- 37 &, 718 \\
8. Falta de afrontamiento & $-1,60$ & 10,23 &,- 61 &, 554 \\
9. Reducción de la tensión & $-6,93$ & 14,62 & $-1,84$ &, 088 \\
10. Acción social &, 33 & 20,40 &, 06 &, 950 \\
11. Ignorar el problema & $-7,00$ & 14,98 & $-1,81$ &, 092 \\
12. Autoinculparse & $-2,67$ & 13,74 &,- 75 &, 465 \\
13. Reservarlo para sí & $-10,33$ & 22,87 & $-1,75$ &, 102 \\
14. Buscar apoyo espiritual & $-4,33$ & 9,42 & $-1,78$ &, 097 \\
15. Fijarse en lo positivo & 3,00 & 11,92 &, 98 &, 346 \\
16. Buscar ayuda profesional & $-5,33$ & 19,77 & $-1,05$ &, 314 \\
17. Buscar diversiones relajantes & $-3,27$ & 17,32 &,- 73 &, 477 \\
18. Distracción física &, 00 & 19,08 &, 00 & 1,000 \\
\hline
\end{tabular}

Nota: ${ }^{*} p \leq, 05 ;{ }^{* *} p \leq, 01 ; * * * p \leq, 001$

Aunque no hay diferencias significativas, mostramos a continuación los resultados más relevantes, es decir, aquellas estrategias en las que las medias medidas al inicio y al final del programa, difieren más entre sí con un $p$-valor más pequeño. En la estrategia "Reducción de la tensión" se encuentran los siguientes resultados $(t(14)=-1.84 p=.088)$. En la estrategia "Ignorar el problema" los resultados también muestran variaciones ligeramente llamativas $(t(14)=-1.81 p=.092)$, al igual que en la estrategia "Buscar apoyo espiritual" $(t(14)=-1.78 p=.097)$.

Pese a que no se han encontrado diferencias significativas, sí consideramos relevante el hecho de que haya aumentado la frecuencia de utilización de estas habilidades por parte del alumnado mediador, ha aumentado con la puesta en marcha del programa. De estas tres, la estrategia Reducción de la tensión (9), se considera que es la que más tiene que ver con las habilidades desarrolladas en el equipo de mediación, puesto que uno de los contenidos que se trabaja en la formación sobre mediación es cómo a la hora de abordar un conflicto, un aspecto clave es gestionar este cuando la intensidad emocional no es tan alta y las partes han dejado pasar un tiempo.

Una vez mostrados los datos obtenidos a través de la Escala de afrontamiento para adolescentes (ACS), analizamos los resultados obtenidos con la escala EHS. Las subescalas de este cuestionario 
evalúan distintas habilidades sociales, asimismo la prueba muestra un Índice Global (Puntuación GLOBAL). Podemos observar en la Tabla 4 cómo, en todas las subescalas medidas al finalizar el curso escolar, el grupo experimental obtiene mayor puntuación que el grupo control. Se encuentran diferencias significativas entre las medias de ambas muestras en dos dimensiones de la escala EHS: Autoexpresión en situaciones sociales $(t(30)=2,16 p=, 039<, 05)$; y el Índice global $(t(30)=2,12 p=, 042<, 05)$. Teniendo ambas un tamaño del efecto mediano $(d \geq, 50)$ sobrepasando la media desviación típica.

Tabla 4.

Resultados de la Prueba t de Student para muestras independientes en la escala EHS.

\begin{tabular}{|c|c|c|c|c|c|c|c|}
\hline & \multicolumn{2}{|c|}{ Gr. Experimental } & \multicolumn{2}{|c|}{ Gr. Control } & \multirow[b]{2}{*}{$t(30)$} & \multirow[b]{2}{*}{$p$} & \multirow[b]{2}{*}{$d$ Cohen } \\
\hline & $\mathrm{M}$ & DT & $\mathrm{M}$ & DT & & & \\
\hline $\begin{array}{l}\text { I. Autoexpresión en } \\
\text { situaciones sociales }\end{array}$ & 26,07 & 5,23 & 22,53 & 4,00 & 2,16 & ,039* & ,76 \\
\hline $\begin{array}{l}\text { II. Defensa de los propios } \\
\text { derechos como consumidor }\end{array}$ & 15,13 & 2,85 & 14,41 & 3,12 & 0,68 &, 502 & ,24 \\
\hline $\begin{array}{l}\text { III. Expresión de enfado o } \\
\text { disconformidad }\end{array}$ & 12,67 & 2,50 & 10,82 & 2,74 & 1,98 & ,057 & ,71 \\
\hline $\begin{array}{l}\text { IV. Decir "no" y cortar } \\
\text { interacciones }\end{array}$ & 18,60 & 2,50 & 17,00 & 3,55 & 1,45 & , 156 &, 52 \\
\hline V. Hacer peticiones & 16,20 & 3,63 & 14,06 & 2,49 & 1,97 & 059 & 69 \\
\hline $\begin{array}{l}\text { VI. Iniciar interacciones } \\
\text { positivas con el sexo opuesto }\end{array}$ & 15,07 & 2,96 & 14,06 & 2,54 & 1,04 & ,308 & 37 \\
\hline Puntuación GLOBAL & 103,73 & 14,80 & 92,88 & 14,08 & 2,12 &, $042 *$ & ,75 \\
\hline
\end{tabular}

Un resultado interesante es que la dimensión en la que aparecen diferencias significativas entre las dos muestras - "Autoexpresión en situaciones sociales" (I)- refleja la capacidad de expresarse de forma espontánea y sin ansiedad en distintos tipos de situaciones sociales. Una alta puntuación en este factor indica que existe facilidad para relacionarse e interacción en contextos diversos, y la capacidad para expresar opiniones y sentimientos. La formación que reciben los estudiantes mediadores incluye aspectos teóricos y prácticos relacionados con esta capacidad de expresarse con los demás de manera asertiva.

Analizamos finalmente los resultados encontrados al comparar las puntuaciones en la escala EHS del grupo experimental entre el momento inicial y el final del programa. El objetivo, en este caso, es conocer si existen cambios en las habilidades sociales desarrolladas por los alumnos mediadores tras su participación en el programa. En la Figura 2 se puede observar cómo todas las estrategias, excepto "Hacer peticiones" son utilizadas más frecuentemente en la evaluación postest. Añadimos en este análisis los datos que ofrece la escala EHS: un baremo compuesto por adolescentes $(\mathrm{N}=1.017)$ de edades aproximadas a las de nuestro estudio (Gismero, 2000). Se puede observar cómo los alumnos mediadores, 
tanto en la evaluación pretest como postest, parecen disponer de mayor número de habilidades sociales en comparación con la muestra estandarizada utilizada en la prueba.

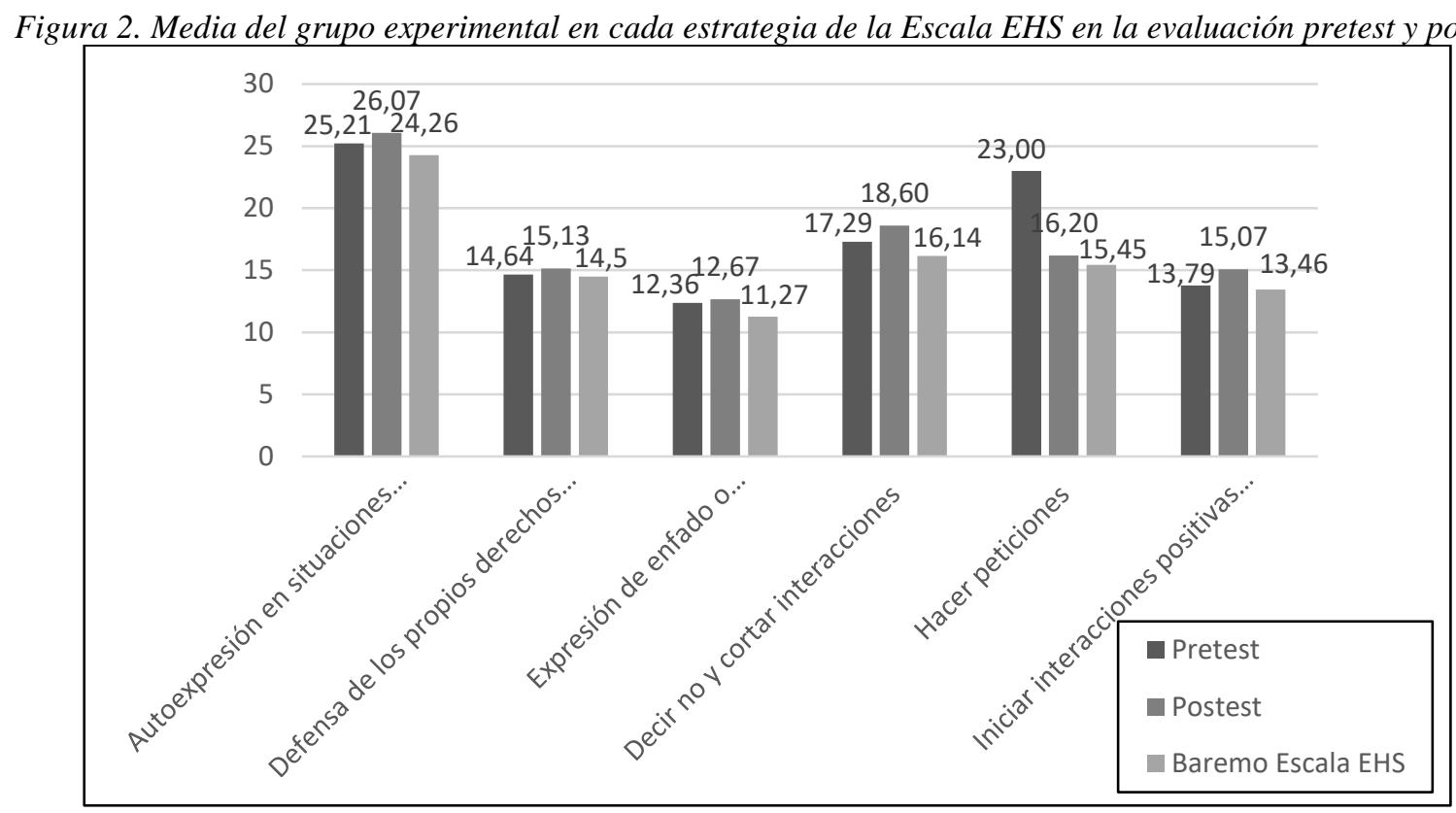

Los resultados muestran que existe una diferencia significativa, medida a través de la $t$ de Student para muestras relacionadas. En la variable Iniciar interacciones positivas con el sexo opuesto (VI), los datos reflejan significatividad estadística $(t(13)=-2.19 p=.047)$ al comparar los valores previos y posteriores al paso por el equipo de mediación. En las Tabla 5 mostramos los resultados obtenidos en la prueba $t$.

Tabla 5.

Resultados de la Prueba T de Student para muestras relacionadas en la escala EHS.

\begin{tabular}{llrrr}
\hline & M & DT & \multicolumn{1}{c}{$t(13)$} & \multicolumn{1}{c}{$p$} \\
\hline I. Autoexpresión en situaciones sociales & $-1,36$ & 4,94 & $-1,03$ &, 323 \\
II. Defensa de los propios derechos como & $-0,71$ & 3,34 & $-0,80$ &, 438 \\
consumidor & & & & \\
III. Expresión de enfado o disconformidad & $-0,57$ & 1,74 & $-1,23$ &, 241 \\
IV. Decir "no" y cortar interacciones & $-1,43$ & 3,18 & $-1,68$ &, 117 \\
V. Hacer peticiones & 6,57 & 30,14 & 0,82 &, 429 \\
VI. Iniciar interacciones positivas con el sexo & $-1,43$ & 2,44 & $-2,19$ &, $047^{*}$ \\
opuesto & & & & \\
Puntuación GLOBAL & $-6,07$ & 12,37 & -1.84 &, 089 \\
\hline
\end{tabular}

Nota: ${ }^{*} p \leq, 05 ;{ }^{* *} p \leq, 01 ; * * * p \leq, 001$.

\section{DISCUSIÓN Y CONCLUSIONES}

Se centra la atención en primer lugar en los datos que nos aporta la Escala ACS (Frydenberg \& Lewis, 1996); la mayor parte de las estrategias mostradas es utilizada de manera más frecuente por el 
grupo experimental si lo comparamos con el grupo control. Particularmente, las estrategias en las que se produce mayor diferencia son: Buscar apoyo social (1), Concentrarse en resolver el problema (2), Buscar ayuda profesional (16) y Buscar diversiones relajantes (17). Al aplicar la prueba $t$ de Student se observa que se producen diferencias significativas y, además, se observa un tamaño del efecto grande.

Las diferencias entre el grupo control y el experimental pueden ser explicadas a través de diversas variables; consideramos que, una explicación plausible, es que los estudiantes mediadores han participado en formaciones sobre mediación y han desarrollado la labor de mediador/a con sus compañeros y esto puede ser una oportunidad de poner en práctica las estrategias evaluadas.

Otro aspecto a tener en cuenta es que, al analizar y comparar los datos de la evaluación previa y final, se observa que el alumnado mediador, antes de su elección como tal, ya disponía de más habilidades y estrategias de resolución de conflictos comparados con el grupo de alumnos y alumnas del grupo control. Por tanto, podríamos destacar que los estudiantes seleccionados entre sus compañeros, aquellos que reciben más votos para convertirse en alumnos/as mediadores, ya disponen, antes de formar parte del equipo de mediación, de mayores estrategias y habilidades sociales. También es cierto el hecho de que, si se comparan las habilidades de resolución de conflictos del grupo de alumnado mediador en evaluación pretest y postest, se observa que la mayoría de habilidades se incrementan; es decir, a final de curso, el grupo experimental, formado por los alumnos mediadores mejora en la mayor parte de habilidades de resolución de conflictos evaluadas.

Una de las conclusiones de nuestro trabajo, es que los datos muestran que los alumnos y alumnas mediadores disponen de un mayor número de habilidades y estrategias de resolución de conflictos antes de comenzar el proceso, y esto les hace más susceptibles de ser seleccionados para formar parte del equipo de mediación. Esto se une al hecho de que formar parte de este tipo de programas proporciona a los jóvenes mayores posibilidades formativas y sociales, puesto que reciben instrucción explícita relacionada con cómo gestionar situaciones sociales, cómo resolver conflictos y además disponen de contextos y situaciones diversas en las que entrenar estas habilidades. En este sentido, compartimos la reflexión de Trianes, Muñoz y Jiménez (1997) que señalan que para aprender habilidades sociales es necesaria la experiencia directa y la educación.

A nivel de implicaciones prácticas de nuestra investigación, se destaca como un aspecto central la vía a través de la que se realiza la selección de los alumnos y alumnas mediadores. En este sentido, los resultados muestran que los estudiantes seleccionados para formar parte del equipo de mediación son alumnos y alumnas que ya han adquirido determinadas de habilidades sociales y estrategias para resolver conflictos, y esto implica que podrán enfrentar sus funciones con una mayor garantía. Se valora, por tanto, como algo central diseñar la selección de alumnos ayudantes a través de un proceso en el que se 
tenga en cuenta las habilidades del alumno o alumna, la percepción que tienen los compañeros, e incluso sus profesores, sobre si pueden ser depositarios de su confianza.

Al analizar las estrategias utilizadas por el grupo control y experimental a través de la EHS (Gismero, 2000) se observa que, en las seis subescalas evaluadas, hay una utilización más frecuente de esas habilidades por parte del grupo experimental; cabe destacar que se han encontrado diferencias significativas en el Índice global del nivel de habilidades sociales o aserción por parte de los alumnos/as mediadores al comparar las puntuaciones del grupo control y experimental. Estos resultados convergen con las investigaciones de Lane-Garon, Yergat \& Kralowec (2012) o Ibarrola-García \& Iriarte (2014a) que encuentran diferencias significativas en cuando a la empatía y la toma de perspectiva de los alumnos y alumnas mediadores comparados con los no mediadores.

Como señala Torrego (2013) este tipo de estructuras generan beneficio, no únicamente para las personas que reciben ayuda, sino particularmente para aquellos que la prestan. Nuestro estudio apunta algo que ya defendía Rogers (1980) hace más de tres décadas, en primer lugar, que las habilidades sociales mejoran gracias a la experiencia y, en segundo lugar, que estas estrategias se aprenden gracias al vínculo estrecho que se crea con un "tutor" que acompaña al alumno. También otros estudios como el de Ibarrola-García e Iriarte (2014a) o Cassinerio y Lane-Garon (2006) ponen en evidencia cómo los programas de mediación producen un incremento de la empatía y la conducta altruista.

El segundo dato relevante es que en la estrategia medida por el EHS: Autoexpresión de situaciones sociales también se encuentran diferencias significativas al comparar el grupo control y el experimental. Una posible explicación de puntuaciones elevadas en este factor que mide la habilidad para interaccionar en distintos contextos, y la capacidad para expresar opiniones y sentimientos, podría tener que ver con el hecho de que la formación recibida por los estudiantes mediadores incluye aspectos teóricos y prácticos relacionados con la capacidad de expresarse con los demás de manera asertiva. Además, el propio proceso de mediar entre dos personas exige poner en práctica estas habilidades.

Otro aspecto relevante es que la propia escala EHS ofrece un baremo de una muestra estandarizada, y nuestro estudio muestra que los alumnos y alumnas mediadores utilizan todas las habilidades medidas en mayor grado que el proporcionado por la prueba. Así, estos datos van en la línea de investigaciones como las de Grau, García-Raga \& López-Martín (2016) o Ibarrola-García \& Iriarte (2014b) que muestran la mejora de habilidades de los alumnos participantes en un programa de mediación.

Si atendemos a los resultados de la evaluación pre y postest, se puede observar que se ha producido una diferencia estadísticamente significativa en una de las habilidades sociales medidas dentro del grupo experimental: Iniciar interacciones positivas con el sexo opuesto (VI). Una posible explicación podría estar en que la participación en equipos de mediación da la oportunidad a los 
estudiantes de compartir espacios y tiempos con personas del otro sexo, pero podría haber otras explicaciones, como el simple hecho de que a lo largo del curso escolar el contacto diario en el aula entre chicos y chicas con el grupo pueda ayudar a que los estudiantes superen algunos miedos o vergüenza de acercarse a personas del sexo opuesto.

Finalizamos el apartado señalando algunas de las limitaciones de nuestro estudio. La utilización de una muestra pequeña dificulta la generalización de los resultados, pero cabe destacar que el muestreo intencional ha sido priorizado por el interés de estudiar en profundidad la evolución del alumnado en un contexto real y natural que nos fuera manejable a los investigadores. También hemos de tener en cuenta la dificultad de comparar centros educativos entre sí, ya que existen numerosas variables a controlar y esto dificulta la realización de inferencias.

Nos parece fundamental seguir realizando estudios en esta línea, y responder así a las demandas establecidas desde el Informe 2016 sobre el Estado Educativo Español (Consejo Escolar del Estado, 2016) de seguir realizando estudios que permitan detectar qué prácticas contribuyen a crear un clima de respeto y trabajo en el centro. 


\section{REFERENCIAS BIBLIOGRÁFICAS}

Avilés, J.M. (2017). Los sistemas de apoyo entre iguales (SAI) y su contribución a la convivencia escolar. Innovación Educativa, 27, 5-18

Avilés Martínez, J.M., \& Alonso Elvira, N. (2017). Sistemas de apoyo en la escuela para el desarrollo de la convivencia y la prevención de la violencia escolar. International Journal of Developmental and Educational Psychology. Revista INFAD de Psicología., 7(1), 257-266. doi: http://dx.doi.org/10.17060/ijodaep.2014.n1.v7.797

Boqué, M.C. (2018). La mediación va a la escuela. Hacia un buen plan de convivencia en el centro. Madrid: Narcea.

Cassinerio, C. \& Lane-Garon, P. (2006). Changing School Climate One Mediator at a Time: Year-One Analysis of a School-Based Mediation Program. Conflict Resolution Quarterly, 23(4), 447-460. doi: 10.1002/crq. 149

Consejo Escolar del Estado (2016). Informe 2016 sobre el estado educativo español. Madrid: Secretaría General Técnica. Centro de Publicaciones. Ministerio de Educación, Cultura y Deporte.

España. Ley 27/2005 de 30 de noviembre, de fomento de la educación y la cultura de la paz. Boletín Oficial del Estado, 1 de diciembre de 2005, núm. 287, pp. 39418-39419.

Frydenberg, E. \& Lewis, R. (1996). Escalas de Afrontamiento para Adolescentes (ACS). Madrid: TEA Ediciones.

García-Raga, L.G., Grau, R. \& López-Martín, R. (2017). Mediation as a Process for the Management of Conflict and the Improvement of Coexistence in Education. Procedia - Social and Behavioral Sciences, 237, 465-470.

García-Raga, L. y López, R. (2011). Convivir en la escuela. Una propuesta para su aprendizaje por competencias. Revista de Educación, 356, 531-555.

García-Raga, L.G., Sanchis, I.C., Mora, A.M., \& Santana, G.R. (2016). Fortalezas y debilidades de la mediación escolar desde la perspectiva del alumnado de educación secundaria. Pedagogía Social. Revista Interuniversitaria, 28, 203-215. doi: http://dx.doi.org/10.7179/PSRI_2016.28.15

Gismero González, E. (2000). Escala de habilidades sociales (EHS). Madrid: TEA Ediciones.

Guiral, I.U., Badenes, L.V., \& Adrián, J.E. (2016). El impacto de los programas de mediación entre iguales para prevenir conductas de acoso escolar en los centros de secundaria. Infancia y Aprendizaje: Journal for the Study of Education and Development, 39(3), 512-527.

Grau, R., García-Raga, L. \& López-Martín, R. (2016). Enseñar y aprender convivencia. Análisis de un programa socioeducativo práctico de mejora de la participación democrática en $2{ }^{\circ}$ ciclo de Educación Infantil. Revista Iberoamericana De Educación, 173-196. https://doi.org/https://doi.org/10.35362/rie71010

Ibarrola-García, S. e Iriarte, C. (2013). Percepción de mejora personal y social en los alumnos mediadores y mediados y profesores mediadores. Campo Abierto, 32(2), 117-143. Recuperado de http://qurriculum.webs.ull.es/0_materiales/articulos/Qurriculum\%2027-2014/Qurriculum\%20272014\%20(1).pdf 
Ibarrola-García, S. \& Iriarte, C. (2014a). Desarrollo de las competencias emocional y sociomoral a través de la mediación escolar entre iguales en educación secundaria. Revista Qurriculum, 27, 9-27.

Ibarrola-García, S. \& Iriarte, C. (2014b). Socio-emotional Empowering through Mediation to Resolve Conflicts in a Civic Way. London Review of Education 12(3), 261-273

Lane-Garon, P., Yergat, J. \& Kralowec, C. (2012). Conflict Resolution Education and Positive Behavioral Support: A Climate of Safety for All Learners. Conflict Resolution Quarterly, 30(2), 197217.

Monjas, M.I. (2010). El desarrollo de habilidades sociales como factor de protección ante el bullying. En J.J. Gázquez y M.C. Pérez-Fuentes (Coords.), La convivencia escolar. Aspectos Psicológicos y Educativos (pp. 261-267). Almería: Editorial GEU.

Ortega-Ruiz, R. \& del Rey, R. (2004). Construir la convivencia. Barcelona: Edebé.

Ortega-Ruiz, R., del Rey, R. \& Casas, J.A. (2016). Evaluar el bullying y el cyberbullying validación española del EBIP-Q y del ECIP-Q. Psicología Educativa, 22, 71-79.

Peñalva, A., López, J.J., Vega, A. \& Satrústegui, C. (2015). Clima escolar y percepciones del profesorado tras la implementación de un programa de convivencia escolar. Estudios sobre Educación, 28, 9-28.

Pinto, M.E.G., Torrego, J.C. \& Martins, A. (2016). Gabinetes de mediação de conflitos: estruturas de pacificação, dinámica e resultados. En A.M. Costa, M.L. Carvalho y L.R. Oliveira (Eds.), Sustentabilidade da mediação social: processos e práticas (pp. 107-118). Braga: Universidade do Minho.

Rogers, C.R. (1980). A Way of being. Boston: Houghton Mifflin.

Silva, I., \& Torrego, J.C. (2017). Percepción del alumnado y profesorado sobre un programa de mediación entre iguales. Qualitative Research in Education, 6(2), 214-238. doi: http://dx.doi.org/10.17583/qre.2017.2713

Torrego, J.C. (Coord.) (2013). La ayuda entre iguales para mejorar la convivencia escolar. Madrid: Narcea.

Trianes, M.V., Muñoz, A. y Jiménez, M. (1997). Competencia social: su educación y tratamiento. Madrid: Pirámide.

Uranga, M. (1997). Experiencias de mediación escolar en Gernika. Aula de Innovación Educativa, 65, 65-68.

Usó, I., Villanueva, L., \& Adrián, J.E. (2016). Impact of peer mediation programmes to prevent bullying behaviours in secondary schools/El impacto de los programas de mediación entre iguales para prevenir conductas de acoso escolar en los centros de secundaria. Infancia y Aprendizaje, 39(3), 499-527. doi: http://dx.doi.org/10.1080/02103702.2016.1189122 